\title{
Intra-Articular Implantation of Stromal Vascular Fraction Plus Platelet Rich Plasma in a Degenerative Meniscal Injury
}

\author{
Kristin Comella ${ }^{\text {a, d}}$, Scott Greenberg ${ }^{b}$, Laura Ross ${ }^{\mathrm{c}}$
}

\begin{abstract}
Regenerative medicine and specifically stem cell therapy is growing in popularity in a variety of orthopedic conditions including meniscal injuries. Stromal vascular fraction (SVF) which is easily obtained from adipose (fat tissue) is a mixture of adipose derived stem cells/ mesenchymal stem cells, pericytes, fibroblasts, progenitor/endothelial cells, and other cells. A mini-lipoaspiration procedure to obtain a small sample of fat can be completed using a cannula under local anesthesia. The SVF is separated from the adipocytes (fat cells) using enzyme digestion and centrifugation. Platelet rich plasma (PRP) from the peripheral blood is a mixture of growth factors and cytokines. We present a case study and to our knowledge first in man with a before and after arthroscopic evaluation showing the repair of a meniscal tear using SVF plus PRP. Adipose tissue $(65 \mathrm{~mL})$ was removed via a mini-lipoaspirate procedure under local anesthesia. Approximately $5 \mathrm{~mL}$ of whole fat was washed and prepared for injection. For the remaining $60 \mathrm{~mL}$ of fat, the adipocytes were removed after enzyme digestion and centrifugation to isolate the SVF. PRP (approximately $4 \mathrm{~mL}$ ) was used to resuspend the cells for an intra-articular injection. The patient received five additional PRP injections of $2.5 \mathrm{~mL}$ at $3-4$ week intervals. The patient was monitored over a 12-month period for safety (including severe adverse events (SAEs) and adverse events) and quality of life parameters. In addition, an arthroscopic evaluation was completed at baseline and 9 months post procedure. There were no reported SAEs or other safety complications during the procedure or the follow-up time period. The patient demonstrated reduction of pain with clearly visible repair on the arthroscopic evaluation. The patient expressed an overall improvement in quality of life and willingness to repeat PRP treatments. Based on the outcomes of this case study, intra-articular injection of SVF plus PRP could be a feasible treatment plan for a patient with chronic meniscus injury.
\end{abstract}

Keywords: Adipose derived stromal/stem cells; Meniscus tear; Mes-

Manuscript submitted May 20, 2018, accepted June 12, 2018

aUS Stem Cell, Inc, 13794 NW 4th Street, Suite 212, Sunrise, FL 33325, USA bMagaziner Center for Wellness, 1907 Greentree Road, Cherry Hill, NJ 08003, USA

'Ross Orthopedics, 300 Creek Crossing Blvd \#307, Hainesport, NJ 08036, USA

${ }^{\mathrm{d} C}$ Corresponding Author: Kristin Comella, US Stem Cell, Inc, 13794 NW 4th Street, Suite 212, Sunrise, FL 33325, USA.

Email: kcomella@us-stemcell.com

doi: https://doi.org/10.14740/jmc3091w enchymal stem cells; Stem cells; Stromal vascular fraction; Platelet rich plasma

\section{Introduction}

Meniscal injuries are the most common knee injury with an average annual rate of incidence of 66 per 100,000 [1]. In the United States, meniscal surgeries are performed on approximately 850,000 patients each year [2]. Arthroscopic surgery for meniscal injuries is a commonly performed procedure. The trend towards arthroscopic debridement of degenerative meniscal tears has been more controversial as of late, with several studies suggesting that this procedure can accelerate the arthritic process in certain circumstances especially in older individuals [3]. There is evidence to suggest that there is no benefit to arthroscopic debridement for certain types of meniscal tears over non-operative sham treatments $[4,5]$. Exercise therapy may accomplish similar or better outcomes as compared to surgery with regards to muscle strength and pain [6].

Mesenchymal/multipotential stem cells or MSCs are being used in a variety of diseases and injuries. MSCs can be collected from many tissues in the body. These cells are believed to promote a healing cascade to repair damaged or aged tissue. MSCs can be expanded/multiplied in culture and can differentiate into several different types of tissue. Because MSCs are thought to stimulate the body's natural healing mechanism, they are currently being considered as an option in various indications $[7,8]$. These cells may stimulate an immunomodulatory or paracrine response and reduce inflammation. MSCs have been reported in the literature to have tremendous tissue repair or regenerative capabilities $[8,9]$. This new paradigm in medicine involves exploiting stem cells and taking advantage of their ability to replicate and promote a cascade of events leading to new tissue formation. MSCs may reduce inflammation and scarring, and promote angiogenesis and healing [10]. A proposed mechanism of action of cellular medicine which includes a complex paracrine activity involving the expression and down regulation of cytokines and growth factors is thought to be responsible for the regeneration of damaged tissues. Caplan et al have described a process in which a pericyte becomes activated and becomes an MSC creating a regenerative microenvironment [11]. The body's microvasculature is coated with pericytes which become activated in an injury 
environment. The pericytes release from the capillaries and microvessels after an injury begin the paracrine effect. This complex signaling process causes the homing of other cells to the area to repair damaged tissue and regeneration occurs.

MSCs can be collected in high numbers from adipose or fat tissue. Adipose derived stem cells (ADSCs) are MSCs collected from fat. Subcutaneous fat containing high amounts of stem cells can be easily isolated from the abdomen or flanks via mini-lipoaspiration [12]. ADSCs, like MSCs, are able to replicate in culture and are multipotential with ability to form bone, cartilage, muscle and fat. These cells express a variety of cytokines and growth factors which are known to be part of the healing cascade and can promote new blood vessel formation. Playing a role in the local inflammation process, this complex signaling can facilitate healing and repair of damaged tissue $[13,14]$.

A stromal vascular fraction (SVF) is a cell population that can be isolated from adipose tissue. Fat is collected via minilipoaspiration and the adipocytes (fat cells) are removed to leave behind a variety of cells including ADSCs and growth factors. In particular, a large percentage of the cells in an SVF express CD34, a protein associated with vasculogenesis and angiogenesis [15]. CD34 protein is found on the surface of pericytes and MSCs. More specifically, cells expressing CD34 help to stabilize endothelial networks in a periendothelial location. Due to the high amount of regenerative stem cells from adipose tissue, SVF may offer a novel therapy option for degenerative disease and tissue damage. SVF has recently been reported in many indications as an out-patient therapy. Fat tissue is a preferred source for stem cells as compared to bone marrow due to the relative higher amounts of MSCs and lower amounts of leukocytes [16]. In addition fat tissue has a network of microvessels and capillaries which are rich in pericytes $[17,18]$.

Injection of SVF may be anti-inflammatory, pro-healing, and anti-scarring. Fat tissue can be easily and safely harvested and SVF can be collected bedside making it an attractive therapeutic option for physicians to offer to their patients [19]. A variety of publications have reported on the injection of SVF including: osteoarthritis [20,-21], degenerative disc disease [22], sclerosis [23], tendinopathy [24], congestive heart failure [25], chronic obstructive pulmonary disease (COPD) [26], radiation necrosis [27], xerostomia [28], psoriasis [29], and multiple sclerosis [30].

Here we report a case study on the use of SVF from fat tissue plus PRP for use in meniscus regeneration. Pak et al reported on the use of adipose stem cells used in single case of meniscus tissue repair [31]. The therapy resulted in repair of tear and reduction of pain. A vast majority of publications to date for meniscal repair using stem cells focus on the use of bone marrow derived cells $[32,33]$. The SVF population includes high amount of MSCs as compared to bone marrow. The isolation of fat tissue under local anesthesia in an outpatient procedure is established as a safe protocol and the collection of an SVF for introduction into a damaged meniscus may offer a new therapeutic approach. Several groups have reported on the use of PRP in meniscal injuries. Betancourt and Murrell reported on a case study with the use of a single PRP injection in a degenerative meniscus lesion with considerable improvement in pain symptoms [34].

\section{Case Report}

\section{Study design}

The presented case study was completed on a 56-year-old male with a chronic meniscus injury. He described a pinching pain with noticeable edema. In addition, his knee was unstable. Two prior MRIs obtained before treatment confirmed a tear of the posterior horn of the medial meniscus. The posterior horn of the medial meniscus showed decreased size with increased degeneration in two MRIs over a 3-year period. Questionable small tears extending to the superior and inferior articular surface are visible. The images also showed irregular fraying of the inferior articular surface of the posterior horn of the medial meniscus. An additional MRI taken 1 month prior to treatment confirmed a new partial-thickness free edge tear of the inner margin of the medial meniscus which was also confirmed with arthroscopic imaging.

The patient provided written informed consent to undergo the experimental clinical protocol. The patient also provided written informed consent to publication of outcomes, images and data. A combination of a $5 \mathrm{~mL}$ whole fat graft and SVF suspended in approximately $4 \mathrm{~mL}$ of platelet rich plasma was administered intra-articularly through a 23-gauge needle. The patient received five follow-up PRP injections $(2.5 \mathrm{~mL})$ at 3 - 4 week intervals after initial injection. The patient was monitored for both adverse events (AEs) and serious adverse events (SAEs). SAEs are defined as any fatal or life-threatening events leading to hospitalizations, or requiring major medical interventions. Basic physical parameters such as blood work, blood pressure, weight/height and pulse were collected at baseline. An arthroscopy evaluation was completed at baseline and 9 months after treatment to determine the extent of the meniscal tear.

\section{Cell preparation and study intervention}

A 30-gauge, 0.5 inch needle was used to administer local numbing followed by a small incision (approximately $3 \mathrm{~mm}$ ) using an 18-gauge Nokor needle. A standard tumescent solution containing $1,000 \mathrm{~mL}$ normal saline, $50 \mathrm{~mL}$ of lidocaine $(2 \%), 8 \mathrm{~mL}$ of sodium bicarbonate $(8.4 \%)$, and $1 \mathrm{~mL}$ of epinephrine $(1 \mathrm{mg} /$ $\mathrm{mL}$ ) was prepared. Using an infiltration cannula, approximately $660 \mathrm{~mL}$ of the tumescent solution was introduced into the subcutaneous fat tissue. A $3 \mathrm{~mm}$ Mercedes cannula connected to a Toomey syringe was used to isolate approximately $65 \mathrm{~mL}$ of fat tissue minus the infranate. Approximately $5 \mathrm{~mL}$ of fat was separated, washed and prepared for direct injection. Using a commercially available collection kit and enzyme (Adipocell ${ }^{\mathrm{TM}}$ Enzyme, US Stem Cell, Inc. Sunrise, FL), the remaining $60 \mathrm{~mL}$ of fat was processed to obtain the SVF.

Using a 22-gauge butterfly needle, whole blood was collected into ACD solution A blood tubes. The autologous whole blood was centrifuged at 1,200 $\mathrm{g}$ for $10 \mathrm{~min}$. The population just above the buffy coat (white ring) was collected into a 10 


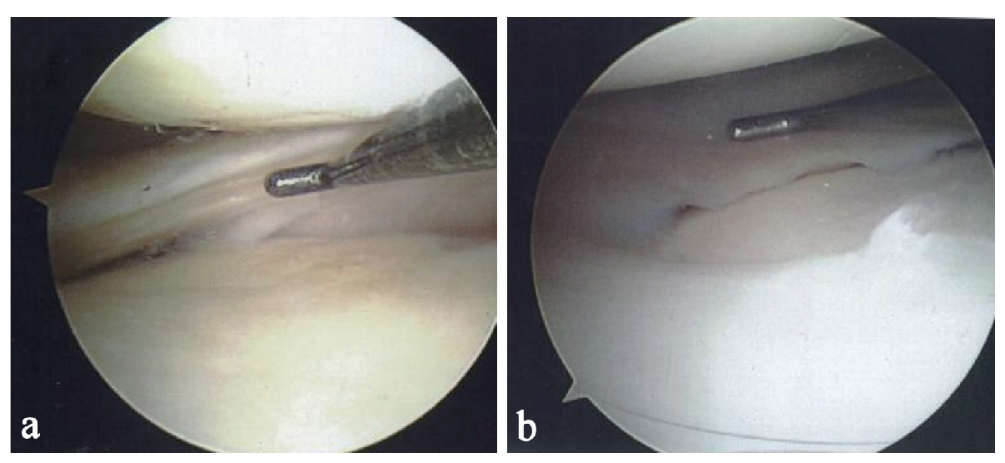

Figure 1. (a) Probe showing tear on the posterior horn of the medial meniscus. (b) Probe showing complete resolution of the meniscal tear.

$\mathrm{mL}$ syringe using a cell extractor. The final SVF pellet was resuspended in $4 \mathrm{~mL}$ of PRP. After saphenous block by $5 \mathrm{~mL}$ $1 \%$ lidocaine and $1 \mathrm{~mL}$ bicarbonate, the $5 \mathrm{~mL}$ whole fat graft was injected intra-articularly proximal to the posterior horn of the meniscus followed by an injection of the SVF and PRP. The patient received five additional PRP injections of $2.5 \mathrm{~mL}$ at 3 - 4 week intervals.

\section{Collection of adipose tissue and cell population}

The mini-lipoaspirate procedure and the intra-articular injection were well tolerated with no AEs reported. From a total of $60 \mathrm{~mL}$ of fat, approximately 30 - 60 million nucleated cells remain in the SVF population after adipocyte depletion. SVF includes a cell population with greater than $50 \%$ CD34 expression and high viability $(>90 \%)$. The ADSCs isolated can also differentiate in culture into fat, bone and cartilage [27].

\section{Patient outcomes}

During the 12-month follow-up period, the patient reported a reduction in pain and an improvement in knee function. Figure 1a shows a tear at baseline on the posterior horn of the medial meniscus. Figure 1b, taken 9 months after the first injection shows complete resolution of the tear. The healed tear could not be penetrated by the probe. The arthroscopic images showing resolution were also consistent with quality of life improvements for the patient including a reduction in pain and resumption of normal activities.

\section{Safety}

The patient did not report AEs or SAEs. The patient experienced very little downtime and was able to resume normal activities in less than 1 week.

\section{Discussion}

One of the most common orthopedic injuries is meniscal inju- ries with arthroscopic debridement frequently performed. Recent literature has exposed the potential limitations of standard of care for degenerative meniscal tears. Orthopedic indications including meniscal injuries have been reported in both preclinical and clinical studies using cellular medicine. The use of SVF in a clinical outpatient setting could be a novel therapy for these conditions. Appropriate protocols (including repeat dosing and dose quantity) for specific indications could be determined with larger sample sizes and placebo controls.

While the scientific community works to understand the complete mechanisms of action during a healing cascade and the role that stem cells play, it is unclear to what extent this therapy may facilitate repair in a meniscal injury. The clinical benefits described in this case study could be due to an immunomodulatory healing effect or as a result of direct cell engraftment. More probably, the observed clinical improvements in the patients are due to complex series of events as a result of various cytokines, chemokines and growth factors that are part of the SVF population. The cascade first reduces inflammation and then stimulates the formation of healthy, new tissue.

This case study demonstrated that intra-articular injection of SVF plus PRP in a knee meniscal injury can be safely completed in an outpatient setting. The procedure was well tolerated with no reported SAEs or AEs. The patient experienced a reduction in pain with an improved quality of life including increased activity. Arthroscopic evaluation showed new tissue formation and resolution of defects. This case study suggested that SVF is a safe and feasible method to address meniscus injuries and future studies could help to establish the long-term effects. Anticipated outcomes could be determined by larger clinical studies. Double blind placebo-controlled studies could address specific dosing and frequency of injections as compared to standard of care interventions.

\section{Declarations}

The patient provided written informed consent and agreed to participate in the study; and agreed to have their data published. The datasets during and/or analysed during the current study are available from the corresponding author on reasonable request. 


\section{Conflict of Interest}

$\mathrm{KC}$ is an officer of US Stem Cell, Inc. US Stem Cell is a publicly traded company providing physician based stem cell therapies to human and animal patients as well as a developer of autologous cell therapies.

\section{Author Contributions}

KC and SG designed the protocol. SG and LR were responsible for clinical procedures and follow-up of patients. $\mathrm{KC}$ wrote the manuscript. All authors contributed toward data analysis, drafting and critically revising the paper and agree to be accountable for all aspects of the work. All authors read and approved the final manuscript.

\section{Abbreviations}

SVF: stromal vascular fraction; ADSCs: adipose derived stem/ stromal cells; MSC: mesenchymal stem cell; SAE: severe adverse event; AE: adverse event; PRP: platelet rich plasma

\section{References}

1. Mordecai SC, Al-Hadithy N, Ware HE, Gupte CM. Treatment of meniscal tears: An evidence based approach. World J Orthop. 2014;5(3):233-241.

2. Arendt EA, ed. Orthopaedic Knowledge Update: Sports Medicine 2. Rosemont, Ill: American Academy of Orthopaedic Surgeons; 1999.

3. Moseley JB, O'Malley K, Petersen NJ, Menke TJ, Brody BA, Kuykendall DH, Hollingsworth JC, et al. A controlled trial of arthroscopic surgery for osteoarthritis of the knee. N Engl J Med. 2002;347(2):81-88.

4. Khan M, Evaniew N, Bedi A, Ayeni OR, Bhandari M. Arthroscopic surgery for degenerative tears of the meniscus: a systematic review and meta-analysis. CMAJ. 2014;186(14):1057-1064.

5. Sihvonen R, Paavola M, Malmivaara A, Itala A, Joukainen A, Nurmi H, Kalske J, et al. Arthroscopic partial meniscectomy versus sham surgery for a degenerative meniscal tear. N Engl J Med. 2013;369(26):2515-2524.

6. Kise NJ, Risberg MA, Stensrud S, Ranstam J, Engebretsen L, Roos EM. Exercise therapy versus arthroscopic partial meniscectomy for degenerative meniscal tear in middle aged patients: randomised controlled trial with two year follow-up. BMJ. 2016;354:i3740.

7. Hematti P, Keating A. Mesenchymal stromal cells in regenerative medicine: a perspective. Mesenchymal Stromal Cells. 2012:3-16.

8. Przybyt E, Harmsen MC. Mesenchymal stem cells: promising for myocardial regeneration? Curr Stem Cell Res Ther. 2013;8(4):270-277.

9. Caplan AI. Mesenchymal Stem Cells: Time to Change the Name! Stem Cells Transl Med. 2017;6(6):1445-1451.
10. Kobolak J, Dinnyes A, Memic A, Khademhosseini A, Mobasheri A. Mesenchymal stem cells: Identification, phenotypic characterization, biological properties and potential for regenerative medicine through biomaterial micro-engineering of their niche. Methods. 2016;99:62-68.

11. Caplan AI, Correa D. The MSC: an injury drugstore. Cell Stem Cell. 2011;9(1):11-15.

12. Minteer D, Marra KG, Rubin JP. Adipose-derived mesenchymal stem cells: biology and potential applications. Adv Biochem Eng Biotechnol. 2013;129:59-71.

13. Gimble JM, Katz AJ, Bunnell BA. Adipose-derived stem cells for regenerative medicine. Circ Res. 2007;100(9):1249-1260.

14. Rehman J, Traktuev D, Li J, Merfeld-Clauss S, TemmGrove CJ, Bovenkerk JE, Pell CL, et al. Secretion of angiogenic and antiapoptotic factors by human adipose stromal cells. Circulation. 2004;109(10):1292-1298.

15. Traktuev DO, Merfeld-Clauss S, Li J, Kolonin M, Arap W, Pasqualini R, Johnstone $\mathrm{BH}$, et al. A population of multipotent CD34-positive adipose stromal cells share pericyte and mesenchymal surface markers, reside in a periendothelial location, and stabilize endothelial networks. Circ Res. 2008;102(1):77-85.

16. Panfilov IA, de Jong R, Takashima S, Duckers HJ. Clinical study using adipose-derived mesenchymal-like stem cells in acute myocardial infarction and heart failure. Methods Mol Biol. 2013;1036:207-212.

17. Jang Y, Koh YG, Choi YJ, Kim SH, Yoon DS, Lee M, Lee JW. Characterization of adipose tissue-derived stromal vascular fraction for clinical application to cartilage regeneration. In Vitro Cell Dev Biol Anim. 2015;51(2):142150.

18. Aust L, Devlin B, Foster SJ, Halvorsen YD, Hicok K, du Laney T, Sen A, et al. Yield of human adipose-derived adult stem cells from liposuction aspirates. Cytotherapy. 2004;6(1):7-14.

19. Comella K, Parlo M, Daly R, Depasquale V, Edgerton E, Mallory P, Schmidt R, et al. Safety analysis of autologous stem cell therapy in a variety of degenerative diseases and injuries using the stromal vascular fraction. J Clin Med Res. 2017;9(11):935-942.

20. Michalek J, Moster R, Lukac L, Proefrock K, Petrasovic M, Rybar J, Capkova M, et al. WITHDRAWN: Autologous adipose tissue-derived stromal vascular fraction cells application in patients with osteoarthritis. Cell Transplant. 2015.

21. Siennicka K, Zolocinska A, Stepien K, Lubina-Dabrowska N, Maciagowska M, Zolocinska E. Adipose-derived cells (Stromal Vascular Fraction) transplanted for orthopedical or neurological purposes: are they safe enough? Stem Cells International. 2016;(2016):1-5.

22. Comella K, Silbert R, Parlo M. Effects of the intradiscal implantation of stromal vascular fraction plus platelet rich plasma in patients with degenerative disc disease. J Transl Med. 2017;15(1):12.

23. Daumas A, Magalon J, Jouve E, Truillet R, Casanova D, Giraudo L, Veran J, et al. Long-term follow-up after autologous adipose-derived stromal vascular fraction injection into fingers in systemic sclerosis patients. Curr Res 
Transl Med. 2017;65(1):40-43.

24. Usuelli FG, Grassi M, Maccario C, Vigano M, Lanfranchi L, Alfieri Montrasio U, de Girolamo L. Intratendinous adipose-derived stromal vascular fraction (SVF) injection provides a safe, efficacious treatment for Achilles tendinopathy: results of a randomized controlled clinical trial at a 6-month follow-up. Knee Surg Sports Traumatol Arthrosc. 2017.

25. Comella K, Parcero J, Bansal H, Perez J, Lopez J, Agrawal A, Ichim T. Effects of the intramyocardial implantation of stromal vascular fraction in patients with chronic ischemic cardiomyopathy. J Transl Med. 2016;14(1):158.

26. Comella K, Blas JAP, Ichim T, Lopez J, Limon J, Moreno RC. Autologous stromal vascular fraction in the intravenous treatment of end-stage chronic obstructive pulmonary disease: a phase I trial of safety and tolerability. J Clin Med Res. 2017;9(8):701-708.

27. Parcero J, Perez J A, Patel A, Ichim T, Gonzalez S, McQuillan S, Comella K. Autologous adipose derived stromal stem cell implantation to resolve critical limb ischemia: case report. Cureus. 2014;6(5):e182.

28. Comella K, Bell W. First-in-man intraglandular implantation of stromal vascular fraction and adipose-derived stem cells plus platelet-rich plasma in irradiation-in- duced gland damage: a case study. Int Med Case Rep J. 2017; 10:295-299.

29. Comella K, Parlo M, Daly R, Dominessy K. First-in-man intravenous implantation of stromal vascular fraction in psoriasis: a case study. Int Med Case Rep J. 2018;11:5964.

30. Riordan NH, Ichim TE, Min WP, Wang H, Solano F, Lara F, Alfaro M, et al. Non-expanded adipose stromal vascular fraction cell therapy for multiple sclerosis. J Transl Med. 2009;7:29.

31. Pak J, Lee JH, Lee SH. Regenerative repair of damaged meniscus with autologous adipose tissue-derived stem cells. Biomed Res Int. 2014;2014:436029.

32. Fisher, James N., et al. The application of stem cells from different tissues to cartilage repair. Stem Cells International. 2017;(2017):1-14.

33. Centeno CJ, Busse D, Kisiday J, Keohan C, Freeman M, Karli D. Regeneration of meniscus cartilage in a knee treated with percutaneously implanted autologous mesenchymal stem cells. Med Hypotheses. 2008;71(6):900908.

34. Betancourt JP, Murrell WD. Leukocyte-poor platelet-rich plasma to treat degenerative meniscal tear: A case report. J Clin Orthop Trauma. 2016;7(Suppl 1):106-109. 